Internat. J. Math. \& Math. Sci.

Vol. 24, No. 4 (2000) 237-249

S016117120000329X

(C) Hindawi Publishing Corp.

\title{
CHARACTERIZATIONS OF OUTER MEASURES ASSOCIATED WITH LATTICE MEASURES
}

\author{
PAO-SHENG HSU
}

(Received 23 April 1999)

\begin{abstract}
Let $v$ be a finite countably subadditive outer measure defined on all subsets of a set $X$, take a collection $\mathbb{C}$ of subsets of $X$ containing $X$ and $\varnothing$, we derive an outer measure $\rho$ using $v$ on sets in $\mathbb{C}$. By applying this general framework on two special cases in which $v=\mu^{\prime \prime}$, one where $\mu \in M_{\sigma}(\mathcal{L})$ and the other where $\mu \in M_{\sigma}\left(\mathcal{\Sigma}_{1}\right), \mathcal{\Sigma}_{1} \subseteq \mathcal{L}_{2}$ being lattices on a set $X$, we obtain new characterizations of the outer measure $\mu^{\prime \prime}$. These yield useful relationships between various set functions including $\mu_{i}, \mu_{j}, \mu^{\prime \prime}$, and $\mu^{\prime}$.
\end{abstract}

Keywords and phrases. Finite countably subadditive outer measures, condition M, lattice measures.

2000 Mathematics Subject Classification. Primary 28A12, $28 \mathrm{C} 15$.

1. Introduction. In this paper, we establish a general framework for the study of outer measures associated with a lattice measure, in particular, of two previously studied outer measures: $\mu^{\prime}$ which is finitely subadditive and $\mu^{\prime \prime}$ which is countably subadditive. Our terminology is consistent with standard usage in [1, 2, 4]; a summary of notations and terminology as well as a brief background is given in Section 2.

Let $v$ be a finite countably subadditive outer measure defined on all subsets of a set $X$, take a collection $\mathbb{C}$ of subsets of $X$ containing $X$ and $\varnothing$, we derive an outer measure $\rho$ using $v$ on sets in $\mathbb{C}$. We examine two special cases: one in which $\mathbb{C}$ is derived from sets related to a lattice $\hat{L}$ of subsets of $X$; another in which there are two lattices $\hat{L}_{1} \subseteq \hat{\mathcal{L}}_{2}$ on $X$ and $\mathbb{C}$ is derived from $\hat{\Sigma}_{2}$. In the former, we take $v=\mu^{\prime \prime}$, where $\mu \in M_{\sigma}(\mathcal{L})$; in the latter $v=\mu^{\prime \prime}$, where $\mu \in M_{\sigma}\left(\Sigma_{1}\right)$. We obtain new characterizations of $\mu^{\prime \prime}$. This is developed in Section 3, which ends with three immediate consequences of this new framework: a shorter proof of an old result on $\mu^{\prime}=\mu^{\prime \prime}$ under the condition that $\mathcal{L}$ is a $\delta$-lattice; letting $\mathbb{C}$ be derived from $\hat{L}_{2}=\mathbf{S}_{\mu^{\prime \prime}}$ in the second case yields $\mu^{\prime \prime}=\left(\mu^{\prime \prime}\right)^{0}$, where the latter is an outer measure previously studied $[2,4]$ and the regularity of $\mu^{\prime \prime}$ for $\mu \in M^{\sigma}\left(\hat{\Sigma}_{1}\right)$.

In Section 4, we define the notion of condition $\mathrm{M}$ on $\mu^{\prime \prime}$ and examine the case of a single lattice to obtain useful inequalities (Theorem 4.7, Corollaries 4.8 and 4.9) to relate various set functions $\mu_{i}, \mu_{j}, \mu^{\prime \prime}$, and $\mu^{\prime}$. We introduce three "regularity"-type conditions on $\mu \in M_{\sigma}(\mathcal{L})$ and study the relationship between them as well as their effect on the equalities of some of the set functions. The inequalities obtained are used to provide alternate proofs on results on equalities of $\mu^{\prime}$ and $\mu^{\prime \prime}$ on either $\mathcal{L}$ or $\mathcal{L}^{\prime}$. Under the condition of normality of $\mathcal{L}$, we also prove how one of these conditions results in the equality of $\mu \in M(\mathcal{L})$ and $\nu \in M_{\sigma}(\mathcal{L})$. 
In Section 5, we examine the case of a pair of lattices on $X$ and introduce six conditions relating to outer measures $\mu^{\prime \prime}$ and $\nu^{\prime \prime}$, where $\mu \in M_{\sigma}\left(\hat{L}_{1}\right)$ and $\nu \in M_{\sigma}\left(\hat{\Sigma}_{2}\right)$, an extension of $\mu$. The relationships between these conditions are studied. Parallel to Section 4, sets of inequalities relating to various set functions (Theorem 5.4 and Corollary 5.5) are obtained and used to show how the conditions introduced in this section result in the equalities of some of the set functions on $\boldsymbol{\Sigma}_{2}^{\prime}$ and how they relate to the conditions introduced in Section 4 on $\mu^{\prime \prime}$ on $\hat{L}_{1}$.

2. Notations and background. On a nonempty set $X$, a lattice $\mathcal{L}$ of subsets of $X$ contains $X$ and $\varnothing$, the algebra generated by $\mathcal{L}$ is denoted by $A(\mathcal{L})$ and the $\sigma$-algebra generated by $\mathcal{\Sigma}$ is denoted by $\sigma(\mathcal{L})$.

LATTICE PROPERTIES. $\mathcal{L}$ is normal if and only if for all $A, B \in \mathcal{L}, A \cap B=\varnothing$, there exist $C$ and $D$ in $\mathcal{L}$ such that

$$
A \subseteq C^{\prime}, \quad B \subset D^{\prime}, \quad \text { and } \quad C^{\prime} \bigcap D^{\prime}=\varnothing,
$$

$\mathcal{L}$ is a $\delta$-lattice if and only if it is closed under countable intersections.

$\hat{\Sigma}_{1}$ and $\hat{\Sigma}_{2}$ are lattices on $X$, where $\hat{\Sigma}_{1} \subseteq \hat{\Sigma}_{2} . \hat{\Sigma}_{1}$ coseparates $\hat{\Sigma}_{2}$ if for $B_{1}$ and $B_{2}$ in $\hat{\Sigma}_{2}$, $B_{1} \cap B_{2}=\varnothing$, there exist $A_{1}$ and $A_{2}$ in $\mathcal{L}_{1}$ such that $B_{1} \subseteq A_{1}^{\prime}, B_{2} \subseteq A_{2}^{\prime}$, and $A_{1}^{\prime} \cap A_{2}^{\prime}=\varnothing$.

We consider the set $M(\mathcal{L})$ of all non-trivial, non-negative, finitely additive bounded measures on $\mathrm{A}(\boldsymbol{\Sigma})$.

MeASURe PROperties. $M_{R}(\mathcal{L})$ is the set of all $\mathcal{L}$-regular measures on $\mathbf{A}(\mathcal{L}): \mu \in$ $M_{R}(\mathcal{L}) \subseteq M(\mathcal{L})$ if and only if for any $A \in \mathrm{A}(\mathcal{L})$,

$$
\mu(A)=\sup \{\mu(L) \mid L \subseteq A, L \in \mathcal{L}\} .
$$

$M_{\sigma}(\mathcal{L})$ is the set of all $\sigma$-smooth measures on $\mathcal{L}: \mu \in M_{\sigma}(\mathcal{L}) \subseteq M(\mathcal{L})$ if and only if for any sequence,

$$
\left\{L_{n}\right\}, \quad L_{n} \in \mathcal{L}, L_{n} \downarrow \varnothing \text {, we have } \lim _{n} \mu\left(L_{n}\right)=0 .
$$

$M^{\sigma}(\mathcal{L})$ is the set of all $\sigma$-smooth measures on $\mathbf{A}(\mathcal{L}): \mu \in M^{\sigma}(\mathcal{L}) \subseteq M(\mathcal{L})$ if and only if for

$$
\left\{A_{n} \in \mathbf{A}(\hat{\Sigma}) \mid n=1,2, \ldots\right\}, \quad A_{n} \downarrow \varnothing, \lim _{n} \mu\left(A_{n}\right)=0 .
$$

Note that $\mu \in M^{\sigma}(\mathcal{L})$ if and only if $\mu$ is countably additive and can be extended uniquely to $\sigma(\mathcal{L})$, and that an $\mathcal{\mathcal { L }}$-regular measure which is $\sigma$-smooth on $\mathcal{\Sigma}$ is $\sigma$-smooth on $\mathrm{A}(\mathcal{L})$. $M_{R}^{\sigma}(\mathcal{L})$ is the set of all $\mathcal{L}$-regular measures which are $\sigma$-smooth on $\mathbf{A}(\mathcal{L}) . M(\sigma, \mathcal{L})$ is the set of all measures strongly $\sigma$-smooth on $\mathcal{\Sigma}: \mu \in M(\sigma, \mathcal{L}) \subseteq M(\mathcal{L})$ if and only if for any

$$
\left\{L_{n} \in \mathcal{L}, n=1,2, \ldots\right\}, \quad L_{n} \downarrow, \bigcap_{n} L_{n} \in \mathcal{L} \text { then } \mu\left(\bigcap_{n} L_{n}\right)=\lim _{n \rightarrow \infty} \mu\left(L_{n}\right) .
$$

Note that if $\mu \in M(\sigma, \mathcal{L})$, then $\mu$ is countably subadditive on $\mathcal{L}^{\prime}$, i.e., for $L_{n} \in \mathcal{L}$ and $\bigcup_{n=1}^{\infty} L_{n}^{\prime} \in \mathcal{L}^{\prime}, \mu\left(\bigcup_{n=1}^{\infty} L_{n}^{\prime}\right) \leq \sum_{n=1}^{\infty} \mu\left(L_{n}^{\prime}\right)$. 
If, in addition, a measure is $0-1$ valued, we say that it belongs to $I(\mathcal{\Sigma})$, replacing $M$ by $I$ in each of the terms above.

For $\mu_{1}, \mu_{2} \in M(\mathcal{L})$, we say that $\mu_{1} \preceq \mu_{2}(\mathcal{L})$ if and only if $\mu_{1}(L) \leq \mu_{2}(L)$ for any $L \in \mathcal{L}$. Note that in the case that $\mu_{1} \preceq \mu_{2}(\mathcal{L})$, we have:

(i) $\mu_{1} \in M_{R}(\hat{L})$ implies that $\mu_{1}=\mu_{2}$;

(ii) $\mu_{2} \in M_{\sigma}(\mathcal{L})$ implies that $\mu_{1} \in M_{\sigma}(\mathcal{L})$.

ASSOCIATED OUTER MEASURES. We summarize some known results (cf. [1]) on two outer measures $\mu^{\prime \prime}$ and $\mu^{\prime}$.

For $E \subseteq X, \mu \in M(\mathcal{L}), \mu^{\prime}(E)=\inf \left\{\mu\left(L^{\prime}\right) \mid E \subset L^{\prime}, L \in \mathcal{L}\right\}$, and $\mu^{\prime \prime}(E)=\inf \left\{\sum_{i} \mu\left(L_{i}^{\prime}\right) \mid\right.$ $\left.E \subset \bigcup_{i} L_{i}^{\prime}, L_{i} \in \mathcal{L}\right\}$. To avoid a trivial $\mu^{\prime \prime}$ in the case of $0-1$ valued measures, we usually assume $\mu \in M_{\sigma}(\mathcal{L})$ when $\mu^{\prime \prime}$ is considered. The set of all $\mu^{\prime \prime}-\left[\mu^{\prime}-\right]$ measurable sets is denoted by $\mathbf{S}_{\mu^{\prime \prime}}\left[\mathbf{S}_{\mu^{\prime}}\right]$ : for $E \subseteq X, E \in \mathbf{S}_{\mu^{\prime \prime}}$ if and only if $\mu^{\prime \prime}(A)=\mu^{\prime \prime}(A \cap E)+\mu^{\prime \prime}\left(A \cap E^{\prime}\right)$ for all $A \subseteq X$. For a finitely subadditive outer measure $\nu, v$ is regular if for any $A \subseteq X$, there exists $E \in \mathbf{S}_{v}$ such that $A \subseteq E$ and $\nu(A)=v(E)$. If $\mu \in I(\mathcal{L})\left[I_{\sigma}(\mathcal{L})\right]$, then $\mu^{\prime}\left[\mu^{\prime \prime}\right]$ is regular. We have the following results.

(1) $\mu^{\prime}(\varnothing)=0 ; \mu^{\prime}$ is monotone and is a finitely subadditive outer measure. $\mathbf{S}_{\mu^{\prime}}$ is an algebra and $\left.\mu^{\prime}\right|_{\boldsymbol{s}^{\prime}}$ is finitely additive measure.

(2) $\mu^{\prime \prime}$ is a countably additive outer measure. $\mathbf{S}_{\mu^{\prime \prime}}$ is a $\sigma$-algebra and $\left.\mu^{\prime \prime}\right|_{\mathbf{S}_{\mu^{\prime \prime}}}$ is countably additive.

(3) In general, we have the following relationships:

$$
\mu \preceq \mu^{\prime}(\mathcal{\Sigma}), \quad \mu=\mu^{\prime}\left(\mathcal{\Sigma}^{\prime}\right), \quad \mu^{\prime \prime} \preceq \mu\left(\mathcal{\Sigma}^{\prime}\right), \quad \mu^{\prime \prime} \preceq \mu^{\prime} .
$$

(4) $\mu \in M_{R}(\mathcal{L})$ if and only if $\mu=\mu^{\prime}(\mathcal{\Sigma})$.

(5) If $\mu \in M_{\sigma}(\mathcal{\Sigma})$, then

(a) $\mu^{\prime \prime}(X)=\mu(X)$;

(b) $\mu \preceq \mu^{\prime \prime}(\mathcal{L})[1]$.

(6) If $\mu \in M(\sigma, \mathcal{L})$, then $\mu^{\prime \prime}=\mu\left(\mathcal{L}^{\prime}\right)$ [4].

(7) If $\mu \in M_{\sigma}(\mathcal{L}), \mu^{\prime \prime}$ is regular and $\mu^{\prime \prime}=\mu\left(\mathcal{\Sigma}^{\prime}\right)$, then $\mu \in M(\sigma, \mathcal{L})$ [4].

(8) If $\mu \in M_{\sigma}(\mathcal{L}), \mu^{\prime \prime}$ is regular and $\mu^{\prime \prime}=\mu(\mathcal{L})$, then $\mu \in M^{\sigma}(\mathcal{L})$ [1].

Using these outer measures, we have two "regularity"-type of conditions on the measure $\mu: M_{w}(\mathcal{L})$ is the set of all weakly regular measures on $\mathbf{A}(\mathcal{L}): \mu \in M_{w}(\mathcal{L}) \subseteq$ $M(\mathcal{L})$ if and only if for any $L \in \mathcal{L}$,

$$
\mu\left(L^{\prime}\right)=\sup \left\{\mu^{\prime}(\tilde{L}) \mid \tilde{L} \subseteq L^{\prime}, \tilde{L} \in \mathcal{L}\right\} .
$$

$M_{v}(\mathcal{L})$ is the set of all vaguely regular measures on $\mathbf{A}(\mathcal{L}): \mu \in M_{v}(\hat{\mathcal{L}}) \subseteq M_{\sigma}(\mathcal{L})$ if and only if for any $L \in \mathcal{L}$,

$$
\mu\left(L^{\prime}\right)=\sup \left\{\mu^{\prime \prime}(\tilde{L}) \mid \tilde{L} \subseteq L^{\prime}, \tilde{L} \in \mathcal{L}\right\} .
$$

In general, $M_{R}(\mathcal{L}) \subseteq M_{w}(\mathcal{\Sigma})$. If $\mathcal{\Sigma}$ is normal, then $M_{R}(\mathcal{\Sigma})=M_{w}(\mathcal{L})$ (cf. [1]).

3. Framework. We consider a finite countably subadditive outer measure $v$ defined on all subsets of a set $X$. Initially, $\mathbb{C}$ is a collection of subsets of $X$ containing $\varnothing$ and $X$. In addition, let $\mathbb{C}$ be closed under countable union. We define a set function $\rho$ 
using $v$ : for $E \subset X$,

$$
\rho(E)=\inf \{v(C), E \subset C \in \mathbb{C}\} .
$$

We show that $\rho$ is an outer measure with $\nu \preceq \rho$ : the definition yields easily that $\rho$ has value zero at the null set, it is monotone and $v \preceq \rho$. To see that it is countably subadditive, suppose $E_{i} \subset X$, for any $\epsilon>0$, we can find $C_{i} \in \mathbb{C}$ such that $v\left(C_{i}\right)<$ $\rho\left(E_{i}\right)+\epsilon / 2^{i}$ and $E_{i} \subset C_{i}$. So $\sum_{1}^{\infty} v\left(C_{i}\right)<\sum_{1}^{\infty} \rho\left(E_{i}\right)+\epsilon$ and $\bigcup_{i} E_{i} \subset \bigcup_{i} C_{i} \in \mathbb{C}$. Since $v$ is countably subadditive and $\rho$ is defined as a lower bound of all $v(C), C \in \mathbb{C}$, we have

$$
\rho\left(\bigcup_{i} E_{i}\right) \leq v\left(\bigcup_{i} C_{i}\right) \leq \sum_{1}^{\infty} v\left(C_{i}\right)<\sum_{1}^{\infty} \rho\left(E_{i}\right)+\epsilon .
$$

By applying this general setting to two special cases, we gain two new perspectives for our applications: one with one lattice $\mathcal{L}$ on $X$; another with two lattices $\hat{L}_{1}$ and $\hat{L}_{2}$ on $X$, with $\hat{\mathcal{L}}_{1} \subset \hat{\mathcal{L}}_{2}$.

Proposition 3.1. Let $\mathbb{C}$ be the collection of all countable unions of sets from $\mathcal{\Sigma}^{\prime}$ and $v=\mu^{\prime \prime}$, where $\mu \in M(\mathcal{L})$. Then $\rho=\mu^{\prime \prime}$ and $\mu^{\prime \prime}(E)=\inf \left\{\mu^{\prime \prime}\left(\bigcup_{1}^{\infty} L_{i}^{\prime}\right), E \subset \bigcup_{1}^{\infty} L_{i}^{\prime}, L_{i} \in \mathcal{L}\right\}$.

Proof. (a) In general, $\mu^{\prime \prime}=\nu \preceq \rho$.

(b) Suppose that there exists $E \subset X$ such that $\mu^{\prime \prime}(E)<\rho(E)$. Then there exists a sequence $\left\{L_{i}\right\} \subset \mathcal{L}$ such that $E \subset \bigcup_{1}^{\infty} L_{i}^{\prime}=C \in \mathbb{C}$ and

$$
\sum_{1}^{\infty} \mu\left(L_{i}^{\prime}\right)<\rho(E) \leq \mu^{\prime \prime}\left(\bigcup_{1}^{\infty} L_{i}^{\prime}\right)\left(E \subset \bigcup_{1}^{\infty} L_{i}^{\prime}\right) \leq \sum_{1}^{\infty} \mu^{\prime \prime}\left(L_{i}^{\prime}\right) \leq \sum_{1}^{\infty} \mu\left(L_{i}^{\prime}\right),
$$

a contradiction. This gives a new characterization of $\mu^{\prime \prime}$.

Proposition 3.2. With $\hat{\Sigma}_{1} \subset \hat{\mathcal{L}}_{2}$, let $\mathbb{C}$ to be all countable unions of sets from $\hat{\mathcal{L}}_{2}^{\prime}$ and $\nu=\mu^{\prime \prime}$, where $\mu \in M\left(\hat{\Sigma}_{1}\right)$. Then $\rho=\mu^{\prime \prime}$ and

$$
\mu^{\prime \prime}(E)=\inf \left\{\mu^{\prime \prime}\left(\bigcup_{1}^{\infty} L_{2_{i}}^{\prime}\right), E \subset \bigcup_{1}^{\infty} L_{2_{i}}^{\prime}, L_{2_{i}} \in \tilde{\Sigma}_{2}\right\} .
$$

Proof. As before, if $E \subset \bigcup_{1}^{\infty} L_{i}^{\prime}=C \in \mathbb{C},\left\{L_{i}^{\prime}\right\} \subset \hat{L}_{2}^{\prime} \subset \mathcal{L}_{1}^{\prime}$, the inequalities hold and the same contradiction is arrived.

These two characterizations of $\mu^{\prime \prime}$ can be shown independently of our new setting and $\rho$ :

(I) By monotonicity and definition of $\mu^{\prime \prime}$, we see that

$$
\mu^{\prime \prime}(E) \leq \inf \left\{\mu^{\prime \prime}\left(\bigcup_{1}^{\infty} L_{i}^{\prime}\right), E \subset \bigcup_{1}^{\infty} L_{i}^{\prime}, L_{i} \in \mathcal{\Sigma}\right\} .
$$

Suppose that it were a strict inequality, there is a sequence $\left\{\tilde{L}_{i}\right\}$ in $\mathcal{L}$ with

$$
\sum_{1}^{\infty} \mu\left(\tilde{L}_{i}^{\prime}\right)<\inf \mu^{\prime \prime}\left(\bigcup_{i} L_{i}^{\prime}\right) \leq \inf \sum_{i=1}^{\infty} \mu^{\prime \prime}\left(L_{i}^{\prime}\right) \leq \sum_{i=1}^{\infty} \mu\left(L_{i}^{\prime}\right),
$$

a contradiction.

(II) The inequality $\mu^{\prime \prime}(E) \leq \inf \left\{\mu^{\prime \prime}\left(\bigcup_{1}^{\infty} L_{2_{i}}^{\prime}\right) E \subset \bigcup_{1}^{\infty} L_{2_{i}}^{\prime}, L_{2_{i}} \in \mathcal{L}_{2}\right\}$ follows from monotonicity and $\mu^{\prime \prime}$ being the infimum. A strict inequality leads to a contradiction as in (I). 
Applications. (1) Using the first new characterization, we can provide a short proof of a previously known result on two outer measures $\mu^{\prime \prime}$ and $\mu^{\prime}$.

THEOREM 3.3. If $\mathcal{\perp}$ is a $\delta$-lattice, $\mu \in M(\sigma, \mathcal{L})$, then $\mu^{\prime \prime}=\mu^{\prime}$.

Proof. (a) In this case, $\mu \preceq \mu^{\prime \prime}\left(\mathcal{L}^{\prime}\right)$ : if $A \in \mathcal{L}^{\prime}, L_{i} \in \mathcal{L}$, and $A \subset \bigcup_{1}^{\infty} L_{i}^{\prime}=\left(\cap L_{i}\right)^{\prime}=$ $L^{\prime} \in \mathcal{L}^{\prime}$, then $\mu(A) \leq \mu\left(L^{\prime}\right) \leq \Sigma_{1}^{\infty} \mu\left(L_{i}^{\prime}\right)$, using $\sigma$-smoothness for the last inequality. So $\mu(A) \leq \mu^{\prime \prime}(A)$. The reverse inequality holds in general.

(b) Now, we have

$$
\begin{aligned}
\mu^{\prime \prime}(E) & =\inf \left\{\mu^{\prime \prime}\left(\bigcup_{1}^{\infty} L_{i}^{\prime}\right), E \subset \bigcup_{1}^{\infty} L_{i}^{\prime}, L_{i} \in \mathcal{L}\right\}=\inf \left\{\mu^{\prime \prime}\left(L^{\prime}\right), E \subset L^{\prime}, L \in \mathcal{L}\right\} \\
& =\inf \left\{\mu\left(L^{\prime}\right), E \subset L^{\prime}, L \in \mathcal{L}\right\}=\mu^{\prime}(E),
\end{aligned}
$$

using (a).

(2) As a special case of Proposition 3.2 above, if we have $\mu \in M_{\sigma}\left(\hat{\Sigma}_{1}\right)$ and $\hat{\Sigma}_{1} \subset \mathbf{S}_{\mu^{\prime \prime}}$, take $\hat{\Sigma}_{2}=\mathbf{S}_{\mu^{\prime \prime}}, \mathbb{C}$ to be the collection of all countable unions of sets from $\mathcal{\Sigma}_{2}^{\prime}$ and $v=\mu^{\prime \prime}$. Then we have $\mu^{\prime \prime}=\left(\mu^{\prime \prime}\right)^{0}$, where in general, for a finitely subadditive outer measure $\lambda$ and $\lambda$-measurable sets $\mathbf{S}_{\lambda}, \lambda^{0}(E)=\inf \left\{\lambda(M), E \subset M, M \in \mathbf{S}_{\lambda}\right\}$ : by Proposition 3.2, we have

$$
\mu^{\prime \prime}(E)=\inf \left\{\mu^{\prime \prime}\left(\bigcup_{1}^{\infty} L_{2_{i}}^{\prime}\right), E \subset \bigcup_{1}^{\infty} L_{2_{i}}^{\prime}, L_{2_{i}} \in \hat{L}_{2}\right\}=\inf \left\{\mu^{\prime \prime}(M), E \subset M, M \in \mathbf{S}_{\mu^{\prime \prime}}\right\}
$$

since $\mathbf{S}_{\mu^{\prime \prime}}$ is a $\sigma$-algebra, $\bigcup_{1}^{\infty} L_{2_{i}}^{\prime}=M \in \mathbf{S}_{\mu^{\prime \prime}}$. This is a special case of a finitely subadditive outer measure previously studied [2, 4]. Since in this case $\mu^{\prime \prime}$ is countably additive, the equality $\mu^{\prime \prime}=\left(\mu^{\prime \prime}\right)^{0}$ is equivalent to $\mu^{\prime \prime}$ being regular [2].

(3) In the classical treatment, with $\mu \in M^{\sigma}(\mathcal{L})$ and the covering class $\mathbb{C}$ of measurable sets generated by the classical outer measure $\mu^{*}, \mu^{*}$ is a regular outer measure. In our setting, if $\mu \in M^{\sigma}\left(\hat{\Sigma}_{1}\right)$ and $\mathbb{C}$ is the collection of all countable unions of sets from $\hat{\Sigma}_{2}^{\prime}, \hat{\Sigma}_{2}=\mathbf{S}_{\mu^{\prime \prime}}$, and $v=\mu^{\prime \prime}$, we obtain the regularity for $\mu^{\prime \prime}$ : for all positive integers $n$, there exists $M_{n} \in \mathbf{S}_{\mu^{\prime \prime}}, E \subset M_{n}$ and $\mu\left(M_{n}\right) \leq \mu^{\prime \prime}(E)+(1 / n)$, we may construct $M_{n} \downarrow \cap M_{n}=M_{0} \in \mathbf{S}_{\mu^{\prime \prime}}$. So $\lim _{n \rightarrow \infty} \mu\left(M_{n}\right) \leq \mu^{\prime \prime}(E)$. Since $\mu \in M^{\sigma}\left(\Sigma_{1}\right), \lim _{n \rightarrow \infty} \mu\left(M_{n}\right)=$ $\mu\left(M_{0}\right)=\mu^{\prime \prime}(E)$.

4. Case of a single lattice: comparing some set functions. Grassi, Knight, and Vlad looked into the effects of various conditions on outer measures. We combine these with additional new conditions. Using our new characterizations in Propositions 3.1 and 3.2, we relate various inner and outer measures by several useful inequalities to obtain results on outer measures.

DEFINITION 4.1. Let $\mu \in M(\mathcal{L})$. For any $E \subseteq X$, the inner measure

$$
\mu_{i}(E)=\sup \{\mu(L), L \subset E, L \in \mathcal{L}\} .
$$

The inner measure $\mu_{i}$ is finitely additive on $\mathcal{\Sigma}^{\prime}$; for properties (cf. [3, 5]).

DEFINITION 4.2. Let $\mu \in M_{\sigma}(\mathcal{\Sigma})$. For any $E \subseteq X$, define the set function

$$
\mu_{j}(E)=\mu(X)-\mu^{\prime \prime}\left(E^{\prime}\right) .
$$

If $\mu^{\prime \prime}$ is a regular, then $\mu_{j}$ is an inner measure [2]. 
Vlad showed that $\mu_{i} \preceq \mu_{j} \preceq \mu^{\prime \prime} \preceq \mu^{\prime}$ [4].

We define the notion of condition $\mathrm{M}$.

DEFinITION 4.3. For $\mu \in M_{\sigma}(\mathcal{\Sigma})$, we say that $\mu^{\prime \prime}$ satisfies condition $\mathrm{M}$ if a necessary and sufficient condition for a set $E \in \mathbf{S}_{\mu^{\prime \prime}}$ is that $\mu^{\prime \prime}(X)=\mu^{\prime \prime}(E)+\mu^{\prime \prime}\left(E^{\prime}\right)$.

As in the classical case, if $\mu^{\prime \prime}$ is a regular outer measure, then $\mu^{\prime \prime}$ satisfies condition M.

On the other hand, the outer measure $\mu^{\prime}$ is not regular in general but always satisfies condition M (see [1]).

Proposition 4.4. (a) If $\mu \in M_{\sigma}(\mathcal{L})$ satisfies condition $M$ and $\mu^{\prime \prime}(E)=\mu_{j}(E)$ for $E \subseteq$ $X$, then $E \in \mathbf{S}_{\mu^{\prime \prime}}$;

(b) If $E \in \mathbf{S}_{\mu^{\prime \prime}}$, then $\mu^{\prime \prime}(E)=\mu_{j}(E)$.

Proof. (a) By assumption,

$$
\mu^{\prime \prime}(E)=\mu_{j}(E)=\mu(X)-\mu^{\prime \prime}\left(E^{\prime}\right)=\mu^{\prime \prime}(X)-\mu^{\prime \prime}\left(E^{\prime}\right)
$$

therefore $E \in \mathbf{S}_{\mu^{\prime \prime}}$ since $\mu$ satisfies condition M.

(b) If $E \in \mathbf{S}_{\mu^{\prime \prime}}$, then

$$
\begin{aligned}
\mu_{j}(E) & =\mu(X)-\mu^{\prime \prime}\left(E^{\prime}\right)=\mu^{\prime \prime}(X)-\mu^{\prime \prime}\left(E^{\prime}\right) \\
& =\mu^{\prime \prime}(E)+\mu^{\prime \prime}\left(E^{\prime}\right)-\mu^{\prime \prime}\left(E^{\prime}\right)=\mu^{\prime \prime}(E) .
\end{aligned}
$$

LEMMA 4.5. If $\mu \in M_{\sigma}(\mathcal{L})$ and $\mu_{j}=\mu(\mathcal{L})$, then $\mu^{\prime \prime}=\mu\left(\mathcal{L}^{\prime}\right)$.

Proof. For $L \in \mathcal{L}$, if $\mu(L)=\mu_{j}(L)=\mu(X)-\mu^{\prime \prime}\left(L^{\prime}\right)$, then

$$
\mu^{\prime \prime}\left(L^{\prime}\right)=\mu(X)-\mu(L)=\mu\left(L^{\prime}\right)
$$

Proposition 4.6. (a) If $\mu \in M(\sigma, \hat{\mathcal{L}})$, then $\mu_{j}=\mu(\hat{\mathcal{L}})$.

(b) If $\mu \in M_{\sigma}(\mathcal{L}), \mu_{j}=\mu(\mathcal{L})$ and $\mu^{\prime \prime}$ is a regular outer measure, then $\mu \in M(\sigma, \mathcal{L})$.

Proof. (a) For $L \in \mathcal{L}, \mu_{j}(L)=\mu(X)-\mu^{\prime \prime}\left(L^{\prime}\right)=\mu(X)-\mu\left(L^{\prime}\right)=\mu(L)$, since $\mu^{\prime \prime}=\mu\left(\mathcal{L}^{\prime}\right)$ if $\mu \in M(\sigma, \mathcal{L})[4]$.

(b) Using the lemma, we have a regular outer measure $\mu^{\prime \prime}$ which is equal to $\mu$ on $\mathcal{L}^{\prime}$, implying that $\mu \in M(\sigma, \hat{\Sigma})$ (see [4]).

Hence for $\mu \in M_{\sigma}(\mathcal{L})$, under regularity of $\mu^{\prime \prime}$, strongly $\sigma$-smoothness is equivalent to $\mu_{j}=\mu(\mathcal{\Sigma})$.

Now, we establish the following sets of inequalities.

TheOrem 4.7. For $\mu \in M_{\sigma}(\mathcal{}), E \subseteq X$, we have:

$$
\mu_{i}(E) \leq \mu_{j}(E) \leq \sup \left\{\mu^{\prime \prime}\left(\bigcup_{1}^{\infty} L_{i}\right), \bigcap L_{i} \subset E, L_{i} \in \mathcal{L}\right\} \leq \mu^{\prime \prime}(E) \leq \mu^{\prime}(E)
$$


Proof. We need only prove the second and third inequalities. We have

$$
\begin{aligned}
\mu_{j}(E) & =\mu(X)-\mu^{\prime \prime}\left(E^{\prime}\right)=\mu(X)-\inf \left\{\sum \mu\left(L_{i}^{\prime}\right), E^{\prime} \subseteq \bigcup L_{i}^{\prime}\right\} \\
& \leq \mu(X)-\inf \left\{\mu^{\prime \prime}\left(\bigcup L_{i}^{\prime}\right)\right\}=\sup \left\{\mu^{\prime \prime}(X)-\mu^{\prime \prime}\left(\bigcup L_{i}^{\prime}\right)\right\} \\
& \leq \sup \left\{\mu^{\prime \prime}\left(X-\bigcup L_{i}^{\prime}\right)\right\}=\sup \left\{\mu^{\prime \prime}\left(\bigcap L_{i}\right), \bigcap L_{i} \subseteq E\right\} \leq \mu^{\prime \prime}(E)
\end{aligned}
$$

which is an upper bound for all such intersections.

COROLLARY 4.8. On $\mathcal{L}^{\prime}$ : for $L \in \hat{\mathcal{L}}$,

$$
\begin{aligned}
\mu_{i}\left(L^{\prime}\right) & \leq \mu_{j}\left(L^{\prime}\right) \leq \sup \left\{\mu^{\prime \prime}\left(\bigcap_{1}^{\infty} L_{i}\right), \bigcap L_{i} \subset L^{\prime}, L_{i} \in \mathcal{L}\right\} \\
& \leq \mu^{\prime \prime}\left(L^{\prime}\right) \leq \mu^{\prime}\left(L^{\prime}\right)=\mu\left(L^{\prime}\right) .
\end{aligned}
$$

Corollary 4.9. (a) $\mu_{i}\left(L^{\prime}\right) \leq \sup \left\{\mu^{\prime \prime}(\tilde{L}), \tilde{L} \subseteq L^{\prime}, \tilde{L} \in \mathcal{L}\right\} \leq \sup \left\{\mu^{\prime \prime}\left(\bigcap_{1}^{\infty} L_{i}\right), \bigcap L_{i} \subset\right.$ $\left.L^{\prime}, L_{i} \in \mathcal{L}\right\} \leq \mu^{\prime \prime}\left(L^{\prime}\right) \leq \mu^{\prime}\left(L^{\prime}\right)=\mu\left(L^{\prime}\right)$.

(b) $\mu_{i}\left(L^{\prime}\right) \leq \sup \left\{\mu^{\prime \prime}(\tilde{L}), \tilde{L} \subseteq L^{\prime}, \tilde{L} \in \mathcal{L}\right\} \leq \sup \left\{\mu^{\prime}(\tilde{L}), \tilde{L} \subseteq L^{\prime}, \tilde{L} \in \mathcal{L}\right\} \leq \mu^{\prime}\left(L^{\prime}\right)=\mu\left(L^{\prime}\right)$.

We consider three more "regularity"-type conditions on $\mu \in M_{\sigma}(\mathcal{\Sigma})$ :

(1) For

$$
L \in \mathcal{L}, \quad \mu\left(L^{\prime}\right)=\sup \left\{\mu^{\prime \prime}\left(\bigcap L_{i}\right), \bigcap L_{i} \subseteq L^{\prime}, L_{i} \in \mathcal{L}\right\}
$$

(2) For

$$
L \in \mathcal{L}, \quad \mu^{\prime \prime}\left(L^{\prime}\right)=\sup \left\{\mu^{\prime \prime}\left(\bigcap L_{i}\right), \bigcap L_{i} \subseteq L^{\prime}, L_{i} \in \mathcal{L}\right\}
$$

(3) For

$$
L \in \mathcal{L}, \quad \mu^{\prime \prime}\left(L^{\prime}\right)=\sup \left\{\mu(\tilde{L}), \tilde{L} \subseteq L^{\prime}, \tilde{L} \in \mathcal{L}\right\} .
$$

Together with the notions of weakly regularity and vaguely regularity, we have the following results, some of which are known, but the proofs here are different due to our general setting.

Proposition 4.10. (a) $\mu \in M_{v}(\hat{\mathcal{L}}) \Rightarrow(4.9)$ holds and $\mu^{\prime \prime}=\mu^{\prime}=\mu\left(\mathcal{L}^{\prime}\right)$.

(b) (4.9) holds $\Rightarrow$ (4.10) holds.

(c) If (4.9) holds and $\mu^{\prime \prime}$ is a regular outer measure, then $\mu \in M(\sigma, \mathcal{L})$ and $\mathcal{L} \subset \mathbf{S}_{\mu^{\prime \prime}}$.

(d) $\mathcal{\Sigma} \subset \mathbf{S}_{\mu^{\prime \prime}} \Rightarrow$ (4.10) holds.

(e) $M_{v}(\hat{\mathcal{L}}) \subseteq M_{w}(\hat{\mathcal{L}})$.

(f) If (4.10) holds, $\mu_{j}\left(L^{\prime}\right)=\sup \left\{\mu^{\prime \prime}\left(\bigcap L_{i}\right), \bigcap L_{i} \subseteq L^{\prime}, L_{i} \in \mathcal{L}\right\}$ for all $L \in \mathcal{L}$ and $\mu^{\prime \prime}$ satisfies condition $M$, then $\mathcal{\perp} \subset \mathbf{S}_{\mu^{\prime \prime}}$.

(g) $\mu \in M_{R}^{\sigma}(\mathcal{L}) \Rightarrow \mu=\mu_{i}=\mu_{j}=\mu^{\prime \prime}=\mu^{\prime}$ on $\mathcal{\Sigma}$.

(h) If (4.11) holds, either $\mu^{\prime \prime}$ is regular or satisfies condition $M$, then $\mathcal{\perp} \subset \mathbf{S}_{\mu^{\prime \prime}}$.

(i) If $\mu \in M_{\sigma}(\mathcal{L}), \mu=\mu^{\prime \prime}(\mathcal{L})$ and $\mu^{\prime \prime}$ is regular, then $\mathcal{L} \subset \mathbf{S}_{\mu^{\prime \prime}}, \mu \in M(\sigma, \mathcal{L})$, and (4.9) holds.

(j) If (4.9) holds and $\mathcal{\Sigma} \subset \mathbf{S}_{\mu^{\prime \prime}}$, then $\mu=\mu^{\prime \prime}(\mathcal{L})$ and hence $\mu \in M^{\sigma}(\mathcal{L})$.

(k) $(4.11) \Rightarrow(4.10)$. 
Proof. Let $L \in \mathcal{L}$ throughout the proof.

(a) Using the inequalities established in Corollary 4.8, we have,

$$
\begin{aligned}
\mu\left(L^{\prime}\right) & =\sup \left\{\mu(\tilde{L}), \tilde{L} \subset L^{\prime}, \tilde{L} \in \mathcal{L}\right\} \\
& \leq \sup \left\{\mu^{\prime \prime}\left(\bigcap_{1}^{\infty} L_{i}\right), \bigcap L_{i} \subset L^{\prime}, L_{i} \in \mathcal{L}\right\} \\
& \leq \mu^{\prime \prime}\left(L^{\prime}\right) \leq \mu^{\prime}\left(L^{\prime}\right) \leq \mu\left(L^{\prime}\right) .
\end{aligned}
$$

(b) Follows from the same set of inequalities.

(c) We have

$$
\mu\left(L^{\prime}\right)=\sup \left\{\mu^{\prime \prime}\left(\bigcap_{i} L_{i}\right), \bigcap_{i} L_{i} \subseteq L^{\prime}, L_{i} \in \mathcal{L}\right\} \leq \mu^{\prime \prime}\left(L^{\prime}\right)=\mu^{\prime}\left(L^{\prime}\right)=\mu\left(L^{\prime}\right) .
$$

Since $\mu=\mu^{\prime \prime}\left(\mathcal{L}^{\prime}\right)$ and $\mu^{\prime \prime}$ is regular, $\mu \in M(\sigma, \hat{\mathcal{L}})$ (see [4]). Also,

$$
\begin{aligned}
\mu_{j}(L) & =\mu(X)-\mu^{\prime \prime}\left(L^{\prime}\right)=\mu(X)-\sup \left\{\mu^{\prime \prime}\left(\bigcap_{i} L_{i}\right), \bigcap_{i} L_{i} \subseteq L^{\prime}\right\} \\
& =\inf \left\{\mu^{\prime \prime}\left(X-\bigcap_{i} L_{i}\right)\right\}=\inf \left\{\mu^{\prime \prime}\left(\bigcup_{i} L_{i}^{\prime}\right), L \subseteq \bigcup_{i} L_{i}^{\prime}\right\}=\mu^{\prime \prime}(L) .
\end{aligned}
$$

Since $\mu^{\prime \prime}$ is regular and therefore satisfies condition M, by Proposition 4.4(a), $L \in \mathbf{S}_{\mu^{\prime \prime}}$. So $\mathcal{L} \subset \mathbf{S}_{\mu^{\prime \prime}}$.

(d) Since $\mathcal{L} \subset \mathbf{S}_{\mu^{\prime \prime}}, \mu^{\prime \prime}\left(L^{\prime}\right) \leq \mu^{\prime \prime}(X)-\mu^{\prime \prime}(L)=\mu_{j}\left(L^{\prime}\right)$. By Corollary 4.8, therefore

$$
\mu_{j}\left(L^{\prime}\right)=\sup \left\{\mu^{\prime \prime}\left(\bigcap_{1}^{\infty} L_{i}\right), \bigcap L_{i} \subset L^{\prime}, L_{i} \in \mathcal{L}\right\}=\mu^{\prime \prime}\left(L^{\prime}\right) .
$$

(e) Using the facts that $\mu^{\prime \prime} \preceq \mu^{\prime}$ in general and $\mu^{\prime}=\mu$ on $\mathcal{L}^{\prime}$ we have

$$
\begin{aligned}
\mu\left(L^{\prime}\right) & =\sup \left\{\mu^{\prime \prime}(\tilde{L}), \tilde{L} \subseteq L^{\prime}, \tilde{L} \in \mathcal{L}\right\} \\
& \leq \sup \left\{\mu^{\prime}(\tilde{L}), \tilde{L} \subseteq L^{\prime}, \tilde{L} \in \mathcal{L}\right\} \\
& \leq \mu^{\prime}\left(L^{\prime}\right)=\mu\left(L^{\prime}\right)
\end{aligned}
$$

yielding equalities and weak regularity.

(f) By assumption,

$$
\mu^{\prime \prime}\left(L^{\prime}\right)=\sup \left\{\mu^{\prime \prime}\left(\bigcap_{i} L_{i}\right), \bigcap_{i} L_{i} \subseteq L^{\prime}, L_{i} \in \mathcal{L}\right\}=\mu_{j}\left(L^{\prime}\right),
$$

so $\mu^{\prime \prime}(X)=\mu^{\prime \prime}(L)+\mu^{\prime \prime}\left(L^{\prime}\right)$, which implies that $\mathcal{L} \subset \mathbf{S}_{\mu^{\prime \prime}}$ since $\mu^{\prime \prime}$ satisfies condition $\mathrm{M}$.

(g) Since $\mu \in M_{R}^{\sigma}(\mathcal{\Sigma}), \mu=\mu^{\prime \prime}=\mu^{\prime}(\mathcal{\Sigma})$. Also, $\mu=\mu_{i}(\mathcal{\Sigma})$. By Corollary 4.8, we have $\mu=\mu_{i}=\mu_{j}=\mu^{\prime \prime}=\mu^{\prime}$ on $\mathcal{L}$.

(h) By assumption and Corollary 4.8, we have

$$
\mu^{\prime \prime}\left(L^{\prime}\right)=\sup \left\{\mu(\tilde{L}), \tilde{L} \subseteq L^{\prime}, \tilde{L} \in \mathcal{L}\right\}=\mu_{i}\left(L^{\prime}\right) \leq \mu_{j}\left(L^{\prime}\right) \leq \mu^{\prime \prime}\left(L^{\prime}\right),
$$


yielding all equalities and $\mu^{\prime \prime}(X)=\mu^{\prime \prime}(L)+\mu^{\prime \prime}\left(L^{\prime}\right)$. Since $\mu^{\prime \prime}$ is regular or satisfies condition $\mathrm{M}, L \in \mathbf{S}_{\mu^{\prime \prime}}$, or $\mathcal{L} \subset \mathbf{S}_{\mu^{\prime \prime}}$.

(i) If $\mu=\mu^{\prime \prime}(\mathcal{L})$, then $\mu=\mu_{i} \preceq \mu_{j} \preceq \mu^{\prime \prime}=\mu(\mathcal{L})$ yielding all equalities. Since $\mu^{\prime \prime}$ is regular, by Proposition 4.6(b), $\mu \in M(\sigma, \mathcal{L})$. Now

$$
\mu_{j}\left(L^{\prime}\right)=\mu(X)-\mu^{\prime \prime}(L)=\mu(X)-\mu(L)=\mu\left(L^{\prime}\right) .
$$

The inequalities in Corollary 4.8 becomes equalities, yielding (4.9).

(j) If (4.9) holds, then $\mu=\mu^{\prime \prime}\left(\mathcal{L}^{\prime}\right)$. By Proposition 4.4(b), $\Sigma \subset \mathbf{S}_{\mu^{\prime \prime}}$ implies that $\mu_{j}=$ $\mu^{\prime \prime}(\mathcal{L})$. Then $\mu^{\prime \prime}(L)=\mu_{j}(L)=\mu(X)-\mu^{\prime \prime}\left(L^{\prime}\right)=\mu(X)-\mu\left(L^{\prime}\right)=\mu(L)$. Since $\mathcal{L} \subset \mathbf{S}_{\mu^{\prime \prime}}$, $A(\mathcal{L}) \subset \mathbf{S}_{\mu^{\prime \prime}}$ and $\left.\mu^{\prime \prime}\right|_{A(\mathcal{L})}=\mu$ is a countably additive measure which is equivalent to $\mu \in M^{\sigma}(\hat{L})$.

(k) The statement follows from Corollary 4.9.

These results provide a new proof of an old theorem.

THEOREM 4.11 (T. Wibisono [6]). (a) If $\mu \in M^{\sigma}(\mathcal{L}), \mu=\mu^{\prime \prime}(\mathcal{L})$ and $\mu^{\prime \prime}$ satisfies condition $M$, then $\mathcal{\Sigma} \subset \mathbf{S}_{\mu^{\prime \prime}}, \mu \in M^{\sigma}(\mathcal{L})$ and $\mu^{\prime \prime}=\mu^{\prime}=\mu\left(\mathcal{L}^{\prime}\right)$.

(b) If $\mu \in M(\sigma, \mathcal{L})$ and $\mathcal{L} \subset \mathbf{S}_{\mu^{\prime \prime}}$, then $\mu=\mu^{\prime \prime}(\mathcal{L})$.

Proof. By Theorem 4.7, for $L \in \mathcal{L}, \mu(L)=\mu_{i}(L) \leq \mu_{j}(L) \leq \mu^{\prime \prime}(L) \leq \mu^{\prime}(L)$. So $\mu=$ $\mu^{\prime \prime}(\mathcal{L})$ implies that $\mu_{j}=\mu^{\prime \prime}(\mathcal{L})$. With $\mu \in M^{\sigma}(\mathcal{L})$ and $\mu^{\prime \prime}$ satisfies condition M, this implies that $\mathcal{\Sigma} \subset \mathrm{S}_{\mu^{\prime \prime}}$ by Proposition 4.4(a). Also, $\mu_{j}\left(L^{\prime}\right)=\mu(X)-\mu^{\prime \prime}(L)=\mu(X)-\mu(L)=$ $\mu\left(L^{\prime}\right)$. Hence by Corollary 4.8 ,

$$
\mu_{j}\left(L^{\prime}\right)=\sup \left\{\mu^{\prime \prime}\left(\bigcap_{1}^{\infty} L_{i}\right), \bigcap_{i} L_{i} \subset L^{\prime}, L_{i} \in \mathcal{L}\right\}=\mu^{\prime \prime}\left(L^{\prime}\right)=\mu^{\prime}\left(L^{\prime}\right)=\mu\left(L^{\prime}\right),
$$

which means that (4.9) holds. Applying Proposition 4.10(j), we get $\mu \in M^{\sigma}(\mathcal{L})$.

(b) If $\mu \in M(\sigma, \mathcal{L})$, then $\mu_{j}=\mu(\mathcal{L})$ by Proposition 4.6(a). By Proposition 4.4(b), $\mathcal{L} \subset \mathbf{S}_{\mu^{\prime \prime}}$ implies that $\mu_{j}=\mu^{\prime \prime}(\mathcal{L})$. Hence $\mu=\mu^{\prime \prime}(\mathcal{L})$.

COROLLARY 4.12. Under the conditions of Theorem 4.11(a), condition (4.9) holds.

THEOREM 4.13. Under the conditions that $\mu \in M(\mathcal{L}), \nu \in M_{\sigma}(\mathcal{L}), \mu \preceq \nu(\mathcal{L}), \mu(X)=$ $\nu(X)$ and $\mathcal{\Sigma}$ is normal, if $\mu^{\prime \prime}$ satisfies condition (4.11), i.e.,

$$
\mu^{\prime \prime}\left(L^{\prime}\right)=\sup \left\{\mu(\tilde{L}), \tilde{L} \subseteq L^{\prime}, \tilde{L} \in \mathcal{\Sigma}\right\}
$$

for $L \in \mathcal{L}$, then $\mu^{\prime \prime}=v^{\prime \prime}\left(\mathcal{\Sigma}^{\prime}\right)$.

Proof. (a) We know that $\mu^{\prime \prime} \succeq v^{\prime \prime}$ in general.

(b) Since $\mathcal{L}$ is normal, for all $\tilde{L} \subseteq L^{\prime}$, there exist $L_{1}, L_{2} \in \mathcal{L}$ such that $\tilde{L} \subseteq L_{1}^{\prime} \subseteq L_{2} \subseteq L^{\prime}$. We have

$$
\mu^{\prime \prime}(\tilde{L}) \leq \mu^{\prime \prime}\left(L_{1}^{\prime}\right) \leq \mu\left(L_{1}^{\prime}\right) \leq \mu\left(L_{2}\right) \leq v\left(L_{2}\right) \leq \nu^{\prime \prime}\left(L_{2}\right) \leq v^{\prime \prime}\left(L^{\prime}\right)
$$

so $\nu^{\prime \prime}\left(L^{\prime}\right)$ is an upper bound for all $\mu^{\prime \prime}(\tilde{L})$. Therefore, $\mu^{\prime \prime}\left(L^{\prime}\right) \leq \nu^{\prime \prime}\left(L^{\prime}\right)$.

5. Case of a pair of lattices: comparing similar set functions. In this section, we have two lattices on $X: \hat{\Sigma}_{1} \subseteq \mathcal{L}_{2}$, we consistently use $A \in \mathcal{L}_{1}$ and $B \in \mathcal{L}_{2}$. 
THEOREM 5.1 (T. Wibisono [6]). If $\mu \in M_{\sigma}\left(\hat{\Sigma}_{1}\right), \mu^{\prime \prime}$ satisfies condition $M$ and

$$
\mu^{\prime \prime}\left(B^{\prime}\right)=\sup \left\{\mu(A), A \subseteq B^{\prime}, A \in \hat{\mathcal{L}}_{1}\right\},
$$

then

(a) $\hat{\Sigma}_{2} \subseteq \mathbf{S}_{\mu^{\prime \prime}}$;

(b) $\left.\mu^{\prime \prime}\right|_{A\left(\mathcal{\Sigma}_{2}\right)} \in M_{R}^{\sigma}\left(\hat{\Sigma}_{2}\right),\left.\mu \preceq \mu^{\prime \prime}\right|_{A\left(\Sigma_{1}\right)}\left(\mathcal{\Sigma}_{1}\right)$, and $\left.\mu^{\prime \prime}\right|_{A\left(\hat{\Sigma}_{1}\right)} \in M_{R}^{\sigma}\left(\hat{\Sigma}_{1}\right)$.

Proof. (a) We have

$$
\mu^{\prime \prime}\left(B^{\prime}\right)=\sup \left\{\mu(A), A \subseteq B^{\prime}, A \in \hat{\mathcal{L}}_{1}\right\}=\mu_{i}\left(B^{\prime}\right)=\mu_{j}\left(B^{\prime}\right)
$$

using Corollary 4.8. Since $\mu^{\prime \prime}$ satisfies condition M, by Proposition 4.4(a), $\hat{\Sigma}_{2} \subseteq \mathbf{S}_{\mu^{\prime \prime}}$.

(b) Since $\mu^{\prime \prime}$ is countably additive on $\mathbf{S}_{\mu^{\prime \prime}}$ and by part (a), $A\left(\hat{\Sigma}_{2}\right) \subseteq \mathbf{S}_{\mu^{\prime \prime}},\left.\mu^{\prime \prime}\right|_{A\left(\mathcal{L}_{2}\right)} \in$ $M^{\sigma}\left(\hat{\Sigma}_{2}\right)$. The inequalities

$$
\mu^{\prime \prime}\left(B^{\prime}\right)=\sup \left\{\mu(A), A \subseteq B^{\prime}, A \in \hat{\mathcal{L}}_{1}\right\} \leq \sup \left\{\mu^{\prime \prime}(A), A \subseteq B^{\prime}, A \in \hat{L}_{1}\right\} \leq \mu^{\prime \prime}\left(B^{\prime}\right)
$$

implies that $\left.\mu^{\prime \prime}\right|_{A\left(\hat{\Sigma}_{2}\right)} \in M_{R}^{\sigma}\left(\hat{\Sigma}_{2}\right)$ and that $\left.\mu^{\prime \prime}\right|_{A\left(\hat{\Sigma}_{1}\right)} \in M_{R}^{\sigma}\left(\hat{\Sigma}_{1}\right)$. Also, $\left.\mu \preceq \mu^{\prime \prime}\right|_{A\left(\hat{\Sigma}_{1}\right)}\left(\hat{\Sigma}_{1}\right)$.

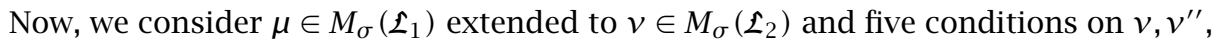
or $\mu^{\prime \prime}$, including the one in Wibisono's theorem: recall that $B \in \hat{\Sigma}_{2}$.

(1) $v\left(B^{\prime}\right)=\sup \left\{v^{\prime \prime}(A), A \subseteq B^{\prime}, A \in \mathcal{L}_{1}\right\}$.

(2) $v\left(B^{\prime}\right)=\sup \left\{v^{\prime \prime}\left(\cap_{1}^{\infty} A_{n}\right), \bigcap_{1}^{\infty} A_{n} \subseteq B^{\prime}, A_{n} \in \mathcal{L}_{1}\right\}$.

(3) $v^{\prime \prime}\left(B^{\prime}\right)=\sup \left\{v^{\prime \prime}\left(\bigcap_{1}^{\infty} A_{n}\right), \bigcap_{1}^{\infty} A_{n} \subseteq B^{\prime}, A_{n} \in \mathcal{L}_{1}\right\}$.

(4) $\nu^{\prime \prime}\left(B^{\prime}\right)=\sup \left\{\mu(A), A \subseteq B^{\prime}, A \in \mathcal{L}_{1}\right\}$.

(5) $\mu^{\prime \prime}\left(B^{\prime}\right)=\sup \left\{\mu(A), A \subseteq B^{\prime}, A \in \mathcal{L}_{1}\right\}$.

We have the following relationships and a result similar to that of Wibisono's.

Proposition 5.2. (a) Condition (1) $\Rightarrow v \in M_{v}\left(\hat{\Sigma}_{2}\right)$.

(b) If $\hat{L}_{1}$ is a $\delta$-lattice, then $(1) \Leftrightarrow(2)$.

(c) If $v \in M\left(\sigma, \hat{\Sigma}_{2}\right)$, then (2) $\Leftrightarrow(3)$.

(d) (2) $\Rightarrow(3)$.

(e) (4) $\Rightarrow(3)$.

(f) $(5) \Rightarrow(4)$.

(g) If (4) holds and $v^{\prime \prime}$ satisfies condition $M$, then $\tilde{\Sigma}_{2} \subseteq \mathbf{S}_{v^{\prime \prime}}$ and $\left.v^{\prime \prime}\right|_{A\left(\mathcal{\Sigma}_{2}\right)} \in M_{R}^{\sigma}\left(\hat{\Sigma}_{2}\right)$. [This is a weaker version of Wibisono's theorem.]

(h) $(5) \Rightarrow v^{\prime \prime}=\mu^{\prime \prime}\left(\hat{\mathcal{L}}_{2}^{\prime}\right)$.

(i) If $\mu^{\prime \prime}\left(B^{\prime}\right)=\sup \left\{\mu^{\prime \prime}(A), A \subseteq B^{\prime}, A \in \mathcal{L}_{1}\right\}$ and $\hat{\Sigma}_{1}$ coseparates $\hat{\Sigma}_{2}$, then $\nu^{\prime \prime}=\mu^{\prime \prime}\left(\mathcal{L}_{2}^{\prime}\right)$.

Proof. (a) Since $\hat{\Sigma}_{1} \subseteq \mathcal{\Sigma}_{2}$,

$$
v\left(B^{\prime}\right)=\sup \left\{v^{\prime \prime}(A), A \subseteq B^{\prime}, A \in \mathcal{L}_{1}\right\} \leq \sup \left\{v^{\prime \prime}(\tilde{L}), \tilde{L} \subseteq B^{\prime}, \tilde{L} \in \mathcal{L}_{2}\right\} .
$$

The reverse inequality is also true because for any $\tilde{L} \subseteq B^{\prime}, \tilde{L} \in \mathcal{L}_{2}, v^{\prime \prime}(\tilde{L}) \leq v^{\prime \prime}\left(B^{\prime}\right) \leq$ $v\left(B^{\prime}\right)$. Hence $v \in M_{v}\left(\hat{\Sigma}_{2}\right)$.

(b) If $\hat{\mathcal{L}}_{1}$ is a $\delta$-lattice, then for $A_{i} \in \hat{\mathcal{L}}_{1}, \bigcap_{1}^{\infty} A_{i}=A \in \hat{\mathcal{L}}_{1}$. Hence (1) $\Leftrightarrow(2)$.

(c) If $v \in M\left(\sigma, \hat{\Sigma}_{2}\right)$, then $v^{\prime \prime}=v\left(\hat{\mathcal{L}}_{2}^{\prime}\right)$. Hence (2) $\Leftrightarrow(3)$. 
(d) By (2),

$$
\begin{aligned}
v\left(B^{\prime}\right) & =\sup \left\{v^{\prime \prime}\left(\bigcap_{1}^{\infty} A_{n}\right), \bigcap_{1}^{\infty} A_{n} \subseteq B^{\prime}, A_{n} \in \Sigma_{1}\right\} \\
& \leq \sup \left\{v^{\prime \prime}\left(\bigcap_{1}^{\infty} B_{n}\right), \bigcap_{1}^{\infty} B_{n} \subseteq B^{\prime}, B_{n} \in \Sigma_{2}\right\} \\
& \leq v^{\prime \prime}\left(B^{\prime}\right) \leq v^{\prime}\left(B^{\prime}\right)=v\left(B^{\prime}\right),
\end{aligned}
$$

the last two inequalities follow from Corollary 4.8. Hence we have equalities and (3) holds.

(e) Since $\hat{\Sigma}_{1} \subseteq \hat{\mathcal{L}}_{2}$, it follows from the definition of the outer measures that $\nu^{\prime \prime} \preceq \mu^{\prime \prime}$. By (4),

$$
\begin{aligned}
v^{\prime \prime}\left(B^{\prime}\right) & =\sup \left\{\mu(A), A \subseteq B^{\prime}, A \in \mathcal{L}_{1}\right\}=\sup \left\{v(A), A \subseteq B^{\prime}, A \in \mathcal{L}_{1}\right\} \\
& \leq \sup \left\{v^{\prime \prime}\left(\bigcap_{1}^{\infty} A_{n}\right), \bigcap_{1}^{\infty} A_{n} \subseteq B^{\prime}, A_{n} \in \mathcal{L}_{1}\right\} \leq v^{\prime \prime}\left(B^{\prime}\right) .
\end{aligned}
$$

The equalities yield (3).

(f) By assumption,

$$
\mu^{\prime \prime}\left(B^{\prime}\right)=\sup \left\{\mu(A), A \subseteq B^{\prime}, A \in \hat{\Sigma}_{1}\right\} \leq \nu^{\prime \prime}\left(B^{\prime}\right)
$$

as above. Hence $v^{\prime \prime}\left(B^{\prime}\right)=\mu^{\prime \prime}\left(B^{\prime}\right)$ since the reverse inequality is true in general; (4) follows.

(g) If

$$
\begin{aligned}
v^{\prime \prime}\left(B^{\prime}\right) & =\sup \left\{\mu(A), A \subseteq B^{\prime}, A \in \mathcal{L}_{1}\right\}=\sup \left\{v(A), A \subseteq B^{\prime}, A \in \mathcal{L}_{1}\right\} \\
& \leq \sup \left\{v(\tilde{B}), \tilde{B} \subseteq B^{\prime}, B \in \mathcal{L}_{2}\right\}=v_{i}\left(B^{\prime}\right) \leq v_{j}\left(B^{\prime}\right) \leq v^{\prime \prime}\left(B^{\prime}\right),
\end{aligned}
$$

resulting in equalities. It follows that $v\left(B^{\prime}\right)+v(B)=v(X)$. Since $v^{\prime \prime}$ satisfies condi-

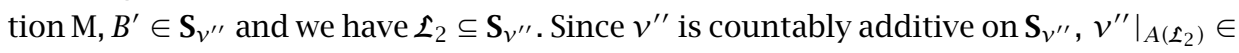
$M^{\sigma}\left(\hat{\Sigma}_{2}\right)$. Also,

$$
v^{\prime \prime}\left(B^{\prime}\right)=\sup \left\{v(\tilde{B}), \tilde{B} \subseteq B^{\prime}, B \in \mathcal{L}_{2}\right\}
$$

for any $B^{\prime} \in \mathcal{L}_{2}$ implies that $\left.\nu^{\prime \prime}\right|_{A\left(\hat{\Sigma}_{2}\right)} \in M_{R}^{\sigma}\left(\hat{\Sigma}_{2}\right)$.

(h) By assumption,

$$
\mu^{\prime \prime}\left(B^{\prime}\right)=\sup \left\{\mu(A), A \subseteq B^{\prime}, A \in \hat{\Sigma}_{1}\right\} \leq v^{\prime \prime}\left(B^{\prime}\right)
$$

as in (e), which implies $v^{\prime \prime}=\mu^{\prime \prime}\left(\boldsymbol{\Sigma}_{2}^{\prime}\right)$.

(i) For each $A \subseteq B^{\prime}$, since $\hat{\Sigma}_{1}$ coseparates $\hat{\Sigma}_{2}$, there exist $A_{1}$ and $A_{2}$ in $\hat{\Sigma}_{1}$ such that $A \subseteq A_{1}^{\prime} \subset A_{2} \subseteq B^{\prime}$. So

$$
\mu^{\prime \prime}(A) \leq \mu^{\prime \prime}\left(A_{1}^{\prime}\right) \leq \mu\left(A_{1}^{\prime}\right)=v\left(A_{1}^{\prime}\right) \leq v\left(A_{2}\right) \leq v^{\prime \prime}\left(A_{2}\right) \leq v^{\prime \prime}\left(B^{\prime}\right) .
$$

Therefore,

$$
\mu^{\prime \prime}\left(B^{\prime}\right)=\sup \left\{\mu(A), A \subseteq B^{\prime}, A \in \hat{\mathcal{L}}_{1}\right\} \leq \nu^{\prime \prime}\left(B^{\prime}\right),
$$

implying $\nu^{\prime \prime}=\mu^{\prime \prime}\left(\mathcal{\Sigma}_{2}^{\prime}\right)$. 
Conclusions in parts (h) and (i) that $\nu^{\prime \prime}=\mu^{\prime \prime}\left(\mathfrak{\Sigma}_{2}^{\prime}\right)$ are useful because if that is true, many of the conditions (1), (2), (3), (4), and (5) as well as others coincide. Related to this is the following condition: in the same setting,

$$
\mu^{\prime \prime}\left(B^{\prime}\right)=\sup \left\{v^{\prime \prime}\left(\bigcap_{1}^{\infty} A_{n}\right), \bigcap_{1}^{\infty} A_{n} \subseteq B^{\prime}, A_{n} \in \mathcal{L}_{1}\right\} .
$$

Proposition 5.3. Condition $(\#) \Rightarrow \nu^{\prime \prime}=\mu^{\prime \prime}\left(\mathfrak{\Sigma}_{2}^{\prime}\right)$ and condition (3).

Proof. If $\bigcap_{1}^{\infty} A_{n} \subseteq B^{\prime}, A_{n} \in \hat{\mathcal{L}}_{1}$ then

$$
\mu^{\prime \prime}\left(B^{\prime}\right)=\sup \left\{v^{\prime \prime}\left(\bigcap_{1}^{\infty} A_{n}\right), \bigcap_{1}^{\infty} A_{n} \subseteq B^{\prime}, A_{n} \in \hat{L}_{1}\right\} \leq v^{\prime \prime}\left(B^{\prime}\right) .
$$

The reverse inequality holds in general. Therefore $v^{\prime \prime}=\mu^{\prime \prime}\left(\boldsymbol{L}_{2}^{\prime}\right)$ and (3) follows.

We also have a set of useful inequalities for the case of a pair of lattices.

THEOREM 5.4. If $\mu \in M_{\sigma}\left(\hat{\mathcal{L}}_{1}\right)$ is extended to $v \in M_{\sigma}\left(\hat{\mathcal{L}}_{2}\right), \hat{\mathcal{L}}_{1} \subseteq \hat{\mathcal{L}}_{2}$, then for an $E \subseteq X$ :

(a) $\mu_{i}(E) \leq \mu_{j}(E) \leq v_{j}(E) \leq \sup \left\{v^{\prime \prime}\left(\bigcap_{n} B_{n}\right), \bigcap_{n} B_{n} \subseteq E, B_{n} \in \hat{\mathcal{L}}_{2}\right\} \leq v^{\prime \prime}(E) \leq v^{\prime}(E)$;

(b) $\mu_{i}(E) \leq \mu_{j}(E) \leq v_{j}(E) \leq \sup \left\{v^{\prime \prime}\left(\bigcap_{n} B_{n}\right), \bigcap_{n} B_{n} \subseteq E, B_{n} \in \hat{\mathcal{L}}_{2}\right\} \leq \nu^{\prime \prime}(E) \leq \mu^{\prime \prime}(E) \leq$ $\mu^{\prime}(E)$;

(c) $\sup \left\{\nu^{\prime \prime}\left(\bigcap_{n} B_{n}\right), \bigcap_{n} B_{n} \subseteq E, B_{n} \in \mathcal{L}_{1}\right\} \leq \sup \left\{\mu^{\prime \prime}\left(\bigcap_{n} B_{n}\right), \bigcap_{n} B_{n} \subseteq E, B_{n} \in \hat{\mathcal{L}}_{2}\right\} \leq$ $\mu^{\prime \prime}(E)$;

(d) $\sup \left\{v^{\prime \prime}\left(\bigcap_{n} A_{n}\right), \bigcap_{n} A_{n} \subseteq E, A_{n} \in \hat{\mathcal{L}}_{1}\right\} \leq \sup \left\{v^{\prime \prime}\left(\bigcap_{n} B_{n}\right), \bigcap_{n} B_{n} \subseteq E, B_{n} \in \hat{\Sigma}_{2}\right\}$;

(e) $\sup \left\{v^{\prime \prime}\left(\bigcap_{n} A_{n}\right), \bigcap_{n} A_{n} \subseteq E, A_{n} \in \mathcal{L}_{1}\right\} \leq \sup \left\{\mu^{\prime \prime}\left(\bigcap_{n} A_{n}\right), \bigcap_{n} A_{n} \subseteq E, A_{n} \in\right.$ $\left.\hat{\Sigma}_{1}\right\} \leq \sup \left\{\mu^{\prime \prime}\left(\bigcap_{n} B_{n}\right), \bigcap_{n} B_{n} \subseteq E, B_{n} \in \hat{\mathcal{L}}_{2}\right\}$.

Proof. The inequalities follow from Theorem 4.7 or $\hat{\Sigma}_{1} \subseteq \hat{\mathcal{L}}_{2}$ or $v^{\prime \prime} \preceq \mu^{\prime \prime}$.

COROLLARY 5.5. For $B \in \hat{\Sigma}_{2}$, we have:

$$
\begin{aligned}
\mu_{i}\left(B^{\prime}\right) & \leq \mu_{j}\left(B^{\prime}\right) \leq \sup \left\{\mu^{\prime \prime}\left(\bigcap_{n} A_{n}\right), \bigcap_{n} A_{n} \subseteq B^{\prime}, A_{n} \in \mathcal{L}_{1}\right\} \\
& \leq \mu^{\prime \prime}\left(B^{\prime}\right) \leq \mu^{\prime}\left(B^{\prime}\right)=\mu\left(B^{\prime}\right) ; \\
\mu_{i}\left(B^{\prime}\right) & \leq \mu_{j}\left(B^{\prime}\right) \leq v_{j}\left(B^{\prime}\right) \leq \sup \left\{v^{\prime \prime}\left(\bigcap_{n} B_{n}\right), \bigcap_{n} B_{n} \subseteq B^{\prime}, B_{n} \in \mathcal{L}_{2}\right\} \\
& \leq v^{\prime \prime}\left(B^{\prime}\right) \leq v^{\prime}\left(B^{\prime}\right)=v\left(B^{\prime}\right) .
\end{aligned}
$$

It follows from the inequalities in Corollary 5.5 that an equality of certain set functions above yields one of the conditions discussed and subsequent consequences.

NoTE 5.6. (a) If $\mu_{i}\left(B^{\prime}\right)=v^{\prime \prime}\left(B^{\prime}\right)$, then we have condition (4).

(b) If $\mu_{i}\left(B^{\prime}\right)=\mu^{\prime \prime}\left(B^{\prime}\right)$, then we have condition (5).

In addition, one of the conditions result in other equalities.

THEOREM 5.7. (a) Condition (4) $\Rightarrow$

$$
\mu_{i}\left(B^{\prime}\right)=\mu_{j}\left(B^{\prime}\right)=v_{j}\left(B^{\prime}\right)=\sup \left\{v^{\prime \prime}\left(\bigcap_{1}^{\infty} B_{n}\right), \bigcap_{1}^{\infty} B_{n} \subseteq B^{\prime}, B_{n} \in \mathcal{L}_{2}\right\}=v^{\prime \prime}\left(B^{\prime}\right)
$$

and $v^{\prime \prime}$ satisfies condition (4.10) on $\hat{\Sigma}_{2}$. 
(b) Condition (5) $\Rightarrow$

$$
\mu_{i}\left(B^{\prime}\right)=\mu_{j}\left(B^{\prime}\right)=\sup \left\{\mu^{\prime \prime}\left(\bigcap_{1}^{\infty} A_{n}\right), \bigcap_{1}^{\infty} A_{n} \subseteq B^{\prime}, A_{n} \in \mathcal{L}_{1}\right\}=\mu^{\prime \prime}\left(B^{\prime}\right)
$$

and $\mu^{\prime \prime}$ satisfies condition (4.10) on $\hat{\Sigma}_{1}$.

(c) Condition (3) $\Rightarrow$

$$
v^{\prime \prime}\left(B^{\prime}\right)=\sup \left\{v^{\prime \prime}\left(\bigcap_{1}^{\infty} B_{n}\right), \bigcap_{1}^{\infty} B_{n} \subseteq B^{\prime}, B_{n} \in \mathcal{L}_{2}\right\}
$$

and $v^{\prime \prime}$ satisfies condition (4.10) on $\hat{\Sigma}_{2}$.

(d) Condition (2) $\Rightarrow$

$$
\sup \left\{v^{\prime \prime}\left(\bigcap_{1}^{\infty} B_{n}\right), \bigcap_{1}^{\infty} B_{n} \subseteq B^{\prime}, B_{n} \in \mathcal{L}_{2}\right\}=v^{\prime \prime}\left(B^{\prime}\right)=v^{\prime}\left(B^{\prime}\right)=v\left(B^{\prime}\right)
$$

and $v^{\prime \prime}$ satisfies condition (4.10) on $\hat{\Sigma}_{2}$.

(e) Condition (\#) $\Rightarrow$

$$
\sup \left\{\mu^{\prime \prime}\left(\bigcap_{1}^{\infty} A_{n}\right), \bigcap_{1}^{\infty} A_{n} \subseteq B^{\prime}, A_{n} \in \hat{L}_{1}\right\}=\mu^{\prime \prime}\left(B^{\prime}\right)=\mu^{\prime}\left(B^{\prime}\right)=\mu\left(B^{\prime}\right)
$$

and $\mu^{\prime \prime}$ satisfies condition (4.10) on $\hat{\Sigma}_{1}$.

Proof. (a) and (b) follow directly from Corollary 5.5; (c) and (d) from Theorem 5.4(d) and the same corollary; (e) from Theorem 5.4(e) and the corollary.

\section{REFERENCES}

[1] P. M. Grassi, Outer measures and associated lattice properties, Int. J. Math. Math. Sci. 16 (1993), no. 4, 687-694. MR 95f:28003. Zbl 778.28009.

[2] J. E. Knight, On finitely subadditive outer measures, J. Math. Sci. (Calcutta) 7 (1996), no. 2, 91-102. MR 98d:28003.

[3] C. Traina, Outer measures associated with lattice measures and their application, Int. J. Math. Math. Sci. 18 (1995), no. 4, 725-734. MR 96g:28019. Zbl 833.28006.

[4] C. Vlad, Outer measures: measurability, $\sigma$-smoothness and regularity, J. Math. Sci. (Calcutta) 7 (1996), no. 2, 75-84. MR 98c:28017.

[5] _ Remarks on finitely subadditive outer measures, Ann. Sci. Math. Québec 20 (1996), no. 1, 93-103. MR 97f:28007. Zbl 863.28003.

[6] T. Wibisono, Measurability and smooth measure extension, Ph.D. thesis, Polytechnic University, New York, 1997, Doctoral Dissertation.

Pao-Sheng Hsu: Department of Mathematics and Statistics, University of Maine, NEVILle Hall, ORONo, MAINE, 04469-5752, USA

E-mail address: hsupao@maine.maine.edu 


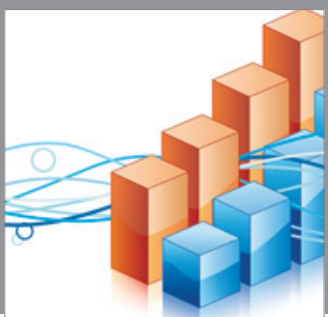

Advances in

Operations Research

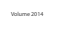

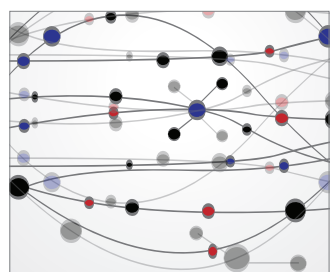

\section{The Scientific} World Journal
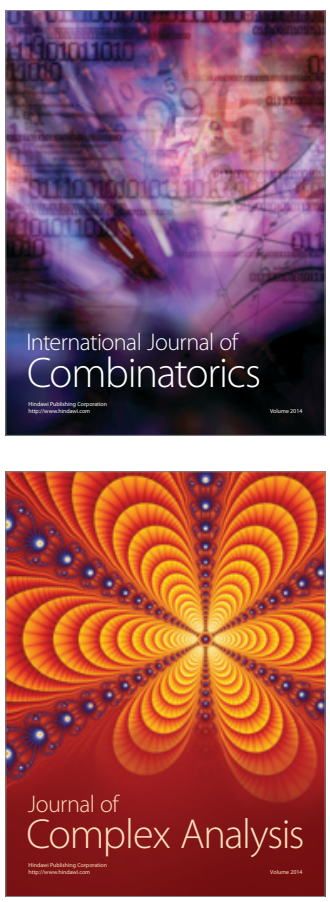

International Journal of

Mathematics and

Mathematical

Sciences
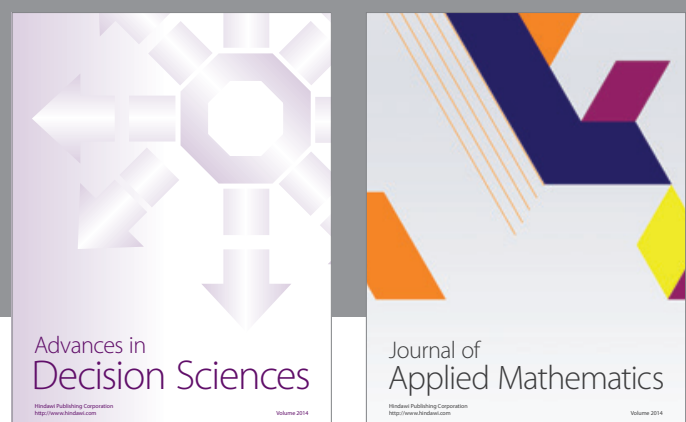

Journal of

Applied Mathematics
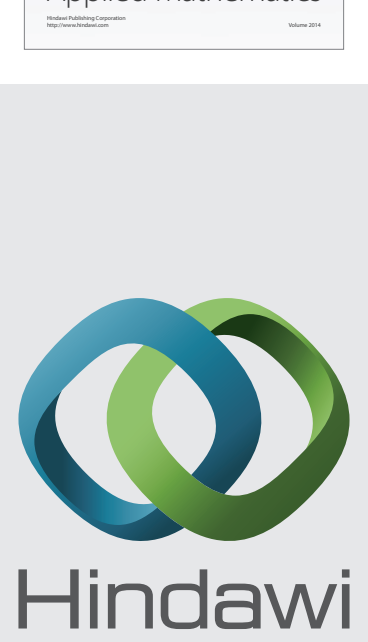

Submit your manuscripts at http://www.hindawi.com
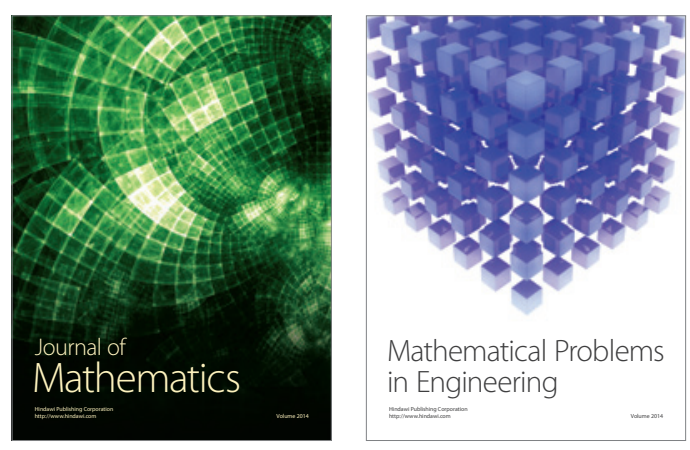

Mathematical Problems in Engineering
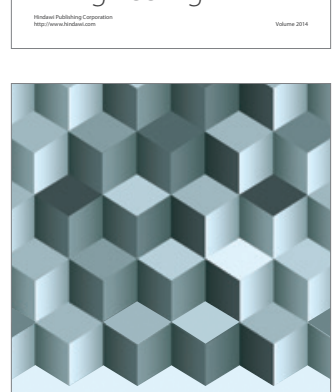

Journal of

Function Spaces
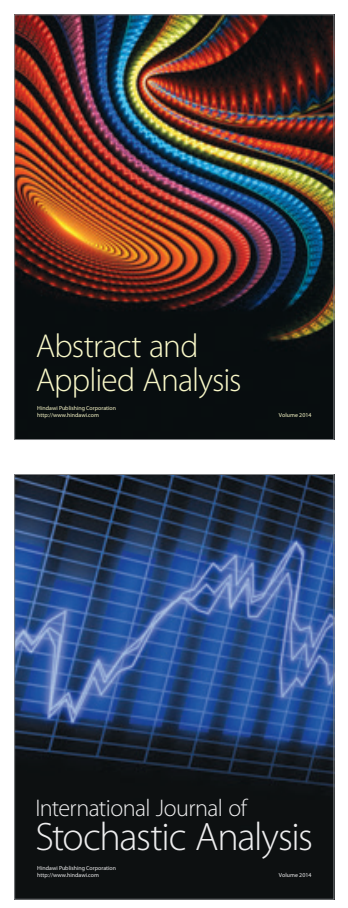

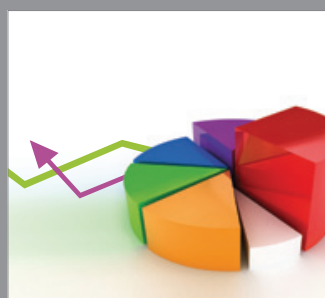

ournal of

Probability and Statistics

Promensencen
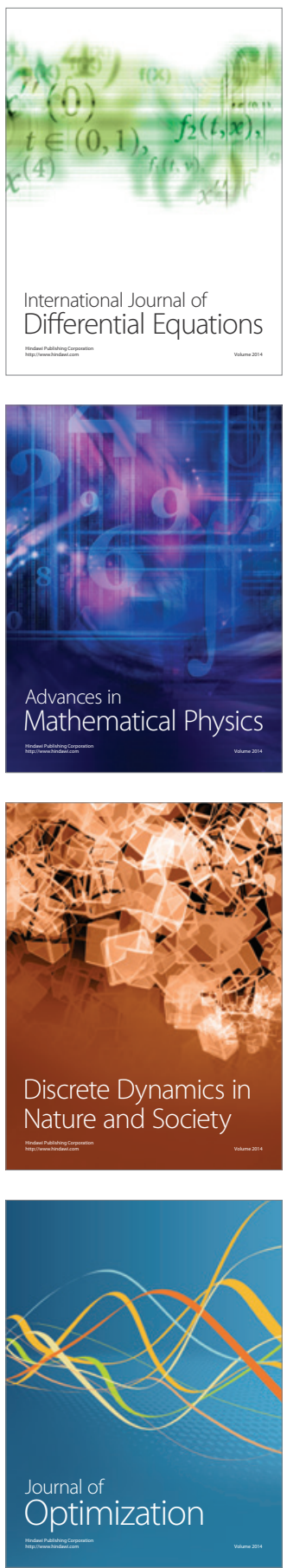